\title{
Group Dynamics in a Security Risk Management Team Context: A Teaching Case Study
}

\author{
Rostyslav Barabanov and Stewart Kowalski \\ Department of Computer and Systems Sciences, Stockholm University, \\ Isafjordsgatan 39, 16440 Kista, Stockholm, Sweden \\ ross.7b@gmail.com, stewart@dsv.su.se
}

\begin{abstract}
This paper discusses the results of a correlation study performed at Stockholm University, which investigated the possible connection between the make-up of security risk management teams and their performance. Three different models were used to construct team composition profiles and were (separately) tested as the seven participating teams completed two practical security risk management tasks. The study has shown that there is a possible correlation between the teams' diversity in terms of the combinations of work related preferences and tendencies (including both risk management specific ones, and non-specific to the risk management context) of their members and the teams' performance.
\end{abstract}

Keywords: Group Dynamics, Security Management, Risk Management, Work Teams.

\section{Introduction}

Risk management research is often based on the assumption that individuals make rational choices without considering how social group dynamics are involved. This paper examines some of the group dynamics factors that can affect teams and individuals in the risk management process. More specifically, the central area under discussion in this paper will be the work related preferences and tendencies of the individuals that make up a security risk management team and their ability to perform effectively.

The study discussed in this paper was carried out at the Department of Computer and Systems Sciences at Stockholm University as part of a Master Thesis project. Space does not allow cover the same scope and level of details in this paper as presented in the original thesis ${ }^{1}$.

The paper is divided into five main sections. Following this short introduction, the motivation for the study will be outlined in brief in section two. Sections three and four describe how the study was set up and carried out and the results it produced respectively. Section five, which is the concluding part of this paper, reflects on the study's findings.

${ }^{1}$ A full copy of the thesis, which provides more in-depth information about the study, can be requested by sending an e-mail to the authors. 


\section{Brief Background}

Teams are widely employed in decision-making activities today [1]. This is not at all surprising bearing in mind that teams not only potentially provide an assortment of complementing abilities that any single individual cannot hope to possess and can additionally offer a set of unique perspectives, which may be instrumental in reaching objective decisions.

A number of studies show that there is a clear connection between the various kinds of diversity of the teams' compositions and team performance [2] [3], greater diversity generally being associated with greater effectiveness, particularly when it comes to creative cognitive tasks.

There can be different kinds of diversity in regards to the composition of teams (e.g. cultural predisposition, skill sets, personality traits or types, ages and gender, etc.). Is a well known practice in security management that diversity is important and can provide the team with an array of complementary abilities and viewpoints [4].

One established and fairly widespread way of modelling team composition in order to better understand and shape team performance is in terms of team roles. A team role can be defined as the teamwork related function a member performs in the team. There are numerous team roles theories (models) in existence; perhaps, the two most widely recognized are Belbin's [5] and Margerison-McCann's [6]; and these models do indeed suggest that the team's diversity (in terms of the team roles typologies put forward in the models) is directly related to the team's performance. These models, however, are not designed to accommodate any specific occupational contexts (e.g. those of security and risk management), raising the question of how (to what extent) universally applicable they are, whether there is a way to complement them in the specific field of interest (security risk management, in this given case), or whether a model that incorporates more context specific factors for profiling teams could be of greater use.

This, in essence, introduces the basic premise for the study to be discussed in the paper. The intention of the study in question was to examine the fairly unexplored connection between the team members' preferences and tendencies that have a conceptually, explicit relation to risk management (e.g. perceptions of risk) and team's effectiveness in that context; and also to put it in contrast with one common and established way to model team composition, in terms of the members' preferences towards certain work functions within the team (i.e. team roles).

The study was built on the notion that greater diversity of these work related preferences and tendencies in team composition is beneficial to team performance. It was also though that the concept of diversity alone may not be sufficiently descriptive of the more and less preferred team member distributions in some cases. Consider a task that may require a combination of diverse viewpoints in order to be completed effectively. In this case certain viewpoints being significantly predominant in the team could potentially defeat the purpose of diversity by suppressing the less represented ones instead of factoring them into the decisions. Thus, it was thought that a certain degree of balance may be necessary in addition to the diversity and so further attention was given to how even the preferences/tendencies were distributed among the team members. 


\section{Design of the Study}

This section consists of three parts. The first part provides an overview of the study; while the second and third parts offer an explanation of how the two main facets of the study, creation of team profiles using different models and theories and testing the performance of the participating teams so that any correlation with the former facet could be examined, were set up and carried out and some of the reasoning that lay behind them.

\subsection{Study Outline}

In order to attain its goals, the study made use of an existing Master level academic course in (corporate) security management. The figure below attempts to illustrate the connection between the said course and the study by means of a simple Input Process - Output model.

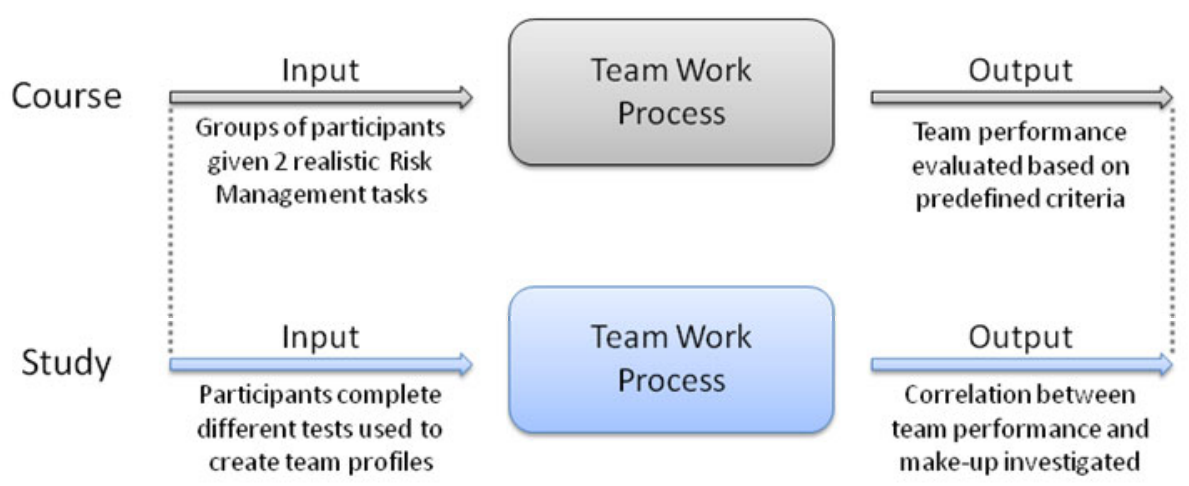

Fig. 1. The variables in the study contrasted by the variables in the course

As can be construed from the above figure, the course provided both the context (i.e. security risk management) and the sample for the experiment, as well as the means for quantifying the sample's performance in that context. While the study itself focused on testing the sample population in order to create team composition profiles with respect to the individual team members' inclinations within the typologies of the chosen models, and quantitatively examining the correlation between the teams' compositions and their performance. It can also be deduced from the figure that the Inputs (the risk management assignments and the tests) were the independent variables in the study and the Outputs (the teams' ranked performance results and their correlation, if any, with the teams' composition) were the dependent variables.

\subsection{Team Modelling}

In total, three different tests were used to create profiles for the teams in terms of their composition. The Cognitive Reflection and Team Roles tests are existing tests that have simply been adopted for the purpose and context of this study; whereas the 
Myths of Nature test has been devised specifically for this study, but using existing theories related to perception of risk. The Team Roles Test was presented in electronic form and completed by the participants online, while the other tests were presented in paper based form.

Cognitive Reflection Test (CRT). This test was formulated by Shane Frederick at the Massachusetts Institute of Technology [7]. The test consists of three seemingly simple questions as shown below:

1) A bat and a ball cost $\$ 1.10$ in total. The bat costs a dollar more than the ball. How much does the ball cost? cents

2) If it takes 5 machines 5 minutes to make 5 widgets, how long would it take 100 machines to make 100 widgets? minutes

3) In a lake, there is a patch of lily pads. Every day, the patch doubles in size. If it takes 48 days for the patch to cover the entire lake, how long would it take for the patch to cover half of the lake? days

The objective of the CRT, as the name implies, is to measure how predisposed the respondents are towards reflecting on the decisions they make before finalizing them. More formally, it can be said that this test measures the respondents' tendency to involve either System 1 or System 2, as they are called by Stanovich and West, cognitive processes in the decision making [8]. System 1 processes being the more quick, unconscious, and intuitive. System 2 processes being the more slow, conscious, and reflective.

In order to demonstrate how this test accomplishes its objective, one of the questions can be used as an example. After examining the first question ("a bat and a ball"), for most individuals the intuitive answer "10 cents" springs to mind, which happens to be incorrect; upon further consideration (reflection), the individual can arrive at the right answer, which is " 5 cents". Likewise, the second and third questions have the intuitive answers "100 minutes" and "24 days", often given, or at least temporarily considered, instead of the correct answers [7], which are "5 minutes" and "47 days" respectively. On close examination, the mathematical problems posed in the questions appear to be very simple, considering the expected target audience for the test. Thus, the outcome of this test is greatly dependent on the respondent's propensity to either rely on the System 1 cognitive processes (settling on the more intuitive, but incorrect solutions) or the System 2 cognitive processes (further reflecting on the problem until the correct solution is found) when attempting to solve the problems, and not so much on their cognitive ability.

The above have direct implications on how individuals perceive and act towards risk. Research shows that the persons who score high (give more accurate answers) on this test tend to have higher risk appetite when dealing with prospective gains and, conversely, lesser risk appetite when dealing with prospective losses in relation to the persons who score low. [7] Hence, the behaviour of the persons scoring low on the test is consistent with the reflection effect suggested by the Prospect Theory [9] and the high scoring persons seem to be inclined towards the contrary.

As could be expected from their tendency to favour System 2 over System 1 processes when making decisions [10], it was also found that the respondents getting 
higher scores on this test are generally more prone to recognize and factor in the longterm implications of their choices, usually preferring the more delayed and larger rewards over the more immediate but smaller ones; and the opposite being true for persons who score low on the test [7].

The test was scored by the number of correct solutions ( 0 to 3 ). The team profile based on the results of this test thus consisted of the combination of the members' scores on the test.

Myths of Nature Test (MNT). The concepts and theories on which the MNT is based on originated in Holling's and Timmerman's research into managed ecosystems [11] [12], where they noted that different managers can adopt different strategies under apparently similar circumstances and that this variance in decision making is associated with their perception of nature and assumptions about how it behaves. The various types of perception encountered were generalized into four "myths of nature".

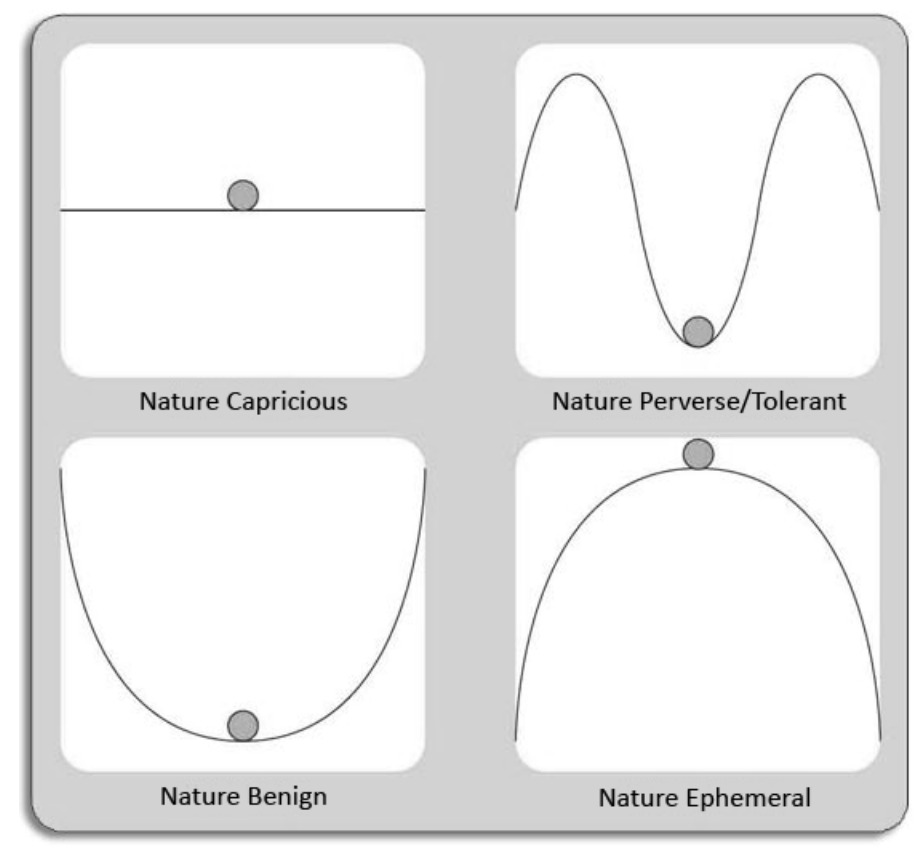

Fig. 2. Graphical representation of the four myths of nature

The four myths of nature were conceptualized in the shape of a ball on different types of landscape as shown on the figure above. The conceptualization can be understood when picturing the consequences of a force being applied to the ball (in undefined trajectory). In Nature Capricious the ball can move in any direction, making this nature unpredictable and any attempts to anticipate or plan for changes in its state futile. Nature Benign suggest that nature is highly predictable, with the ball always ending up at the bottom of the basin, and also very robust since, regardless of the amount of force applied, equilibrium will be restored; thus, although future events can 
be predicted, there is little (if any) need for them to be managed. In the case of Nature Ephemeral, nature is deemed fragile and unsecure, with a careless act potentially having disastrous consequences (balance can be easily lost resulting in the ball falling off), implying extreme care must be taken in managing the nature. The remaining nature, Nature Perverse/Tolerant, assumes that there are limitations to the nature's robustness and predictability, suggesting that discernment of those limits and appropriate regulation are required to maintain stability (prevent the ball from getting knocked over the rim).

Since the perception of nature in this context can be associated with the perception of and, consequently, approach to uncertainty, it is also related to the perception of, and approach to unknown risk thus each myth of nature can be connected to a distinct managerial style being applicable to the domain of risk management. The myths have been linked to the four types of social relationships identified in the Cultural Theory of risk [13] [14]. Nature Capricious subscribers being the Fatalists, Nature Benign Individualists, Nature Ephemeral - Egalitarians, and Nature Perverse/Tolerant being the Hierarchists. One can also deduce that the "natures" assume different risk appetites, Capricious - Risk Neutral, Benign - Risk Seeking, Ephemeral - Risk Averse, and Perverse/Tolerant - tolerant of some risks but not all.

The study participants were presented with both the graphical representation and a textual explanation of the Natures. An attempt was made to make the description of the Myths concise and non-suggestive (and the names of the Myths were replaced with numbers 1 through 4). The textual description of the Myths given to the study participants (but with numbers instead of the original names) was the following:

- Capricious - largely unpredictable; an action could have completely unforeseen results. It's impossible to tell how the situation will play out in the long run.

- Perverse/Tolerant - robust and predictable within certain limitations; forcing the nature beyond those limitations could have severe consequences.

- Benign - largely robust; it tends to reinstate equilibrium over time by itself. Actions have consequences but in the long run the balance is maintained.

- Ephemeral - largely fragile; it must be treated with care because an action could easily upset the balance and have severe consequences.

The study participants were asked to write down which one of the four Myths they thought to be the most agreeable. Thus, the team profile based on the results of this test consisted of the combination of the members' Myths preferences.

Team Roles Test (TRT). The Team Roles model used in this study was developed by Psychtests, a subsidiary of Plumeus Inc., which is a high-tech company based in Montreal, Canada, that specializes in psychological test development and related products and services. Further information about the company can be obtained from their website (http://www.psychtests.com). A statement of compliance with the APA (American Psychological Association) standards for educational and psychological testing is likewise available on Psychtests's website [15].

Similarly to other existing Team Roles models, (e.g. Belbin's [5] or MargerisonMcCann's [6]) the TRT used in the study suggests that the ways in which persons generally contribute to the team or the roles that they tend to assume in a team, can be 
categorized into certain types. In the case of this particular test, it is suggested that there are ten distinct team role types, which are as follows:

- Brainstormer - Imaginative and ambitious, they are excellent at generating ideas (although, they often do not consider practicality thereof).

- Cheerleader - Strive for collaboration and agreement in the team and tend to be keen on motivating team members and keeping the morale up.

- Coordinator - Good organizers; they have a propensity to keep track of resources (human, physical, temporal, etc.), prioritize, and distribute them appropriately.

- Go Getter - Highly motivated and guided by strong personal values, they are very enthusiastic about achieving set goals and inspiring others towards that end.

- Networker - Above all, these individuals contribute to the work by gathering and dispersing useful information through social means in and outside the team.

- Peacemaker - Good at preventing and resolving conflict, they strive for harmony and facilitate continuation of synergy in the team.

- Questioner - Objective, sceptical and insightful, they are rather inquisitive about reasoning behind decisions and are good at providing an alternative viewpoint.

- Team Worker - Ideal team players; they are team oriented and well motivated to do their part and help their team mates to theirs.

- Thinker - Good at analysis and planning, Thinkers often contribute to the team by devising practical solutions out of good but indistinct ideas.

- Verifier - Mainly distinguished by their upmost attention to detail and tendency to maintain proper procedures, they provide quality control for the team.

Team Roles theories are generally based on Jung's theory of Psychological Types [16]. Team roles characterize behaviour, while personality traits and types can influence behaviour but are not types of behaviour themselves. Another notable distinction between team roles and personality traits and types is that team roles are subject to change due to both internal (e.g. a person's preferences can change over time) and external (e.g. expectations and requirements of a certain work assignment, rather than personal preferences, may dictate which functions need to be filled) factors, while personality traits/types are known to be largely stable.

TRT was presented to the study participants in electronic form, consisting of 40 multiple choice questions. Scoring was automatic and was provided by Psychtests. The test measures the person's affinity for each role type on a scale of 1 to 100; the higher the score, the higher the affinity. Therefore, the role type for which the highest score is attained is the one that the person is best suited for and most likely to take on (given the choice). However, Team Roles theories generally state that people can and will assume different team roles as may be required by the circumstances. Based on the size of the teams in the study and the number of team role types in the selected typology, it was decided to take into account each respondent's three highest test scores when creating team profiles; however, the person was assumed to only be able to take on 2 out of the 3 team roles, their absolute highest score and one of the other two, at one given time (as to avoid scenarios where one team member could be potentially undertaking three roles and another one potentially taking on only one). 


\subsection{Team Performance Testing}

The sample in the study consisted of seven groups. The groups were arranged in a semi-random fashion, before the participants took any of the tests featured in the study. The groups were originally comprised of 7 to 8 members each but, due to drop outs, came to be more varied in size (5 to 8 members), which was not damaging to the purpose of this study. Participants were dominantly male, in their mid twenties. Given the fact that the course was open to international/exchange students, the cultural variation was high. Leadership positions were assigned to certain group members, however, the groups were allowed to later rearrange their "administrative structure" by consensus of the members (allowing members the freedom to assume the roles according to their preferences and inclinations).

A large part of the course this study was connected to encompassed an introduction of the participants to The OCTAVE Approach [4], which they were then instructed to use as the framework for completion of the assignments. Ranking criteria used for evaluating team performance was defined prior to the completion of assignments and the actual ranking was carried out by the teacher on the course.

The two practical assignments given to the participants on the course were devised in collaboration with representatives from the global information security company Integralis and the power company Vattenfall, their brief description follows:

- Assignment 1 - The CISO of Vattenfall, subsequently to having been contacted by Integralis regarding the possibility of outsourcing MSS to them, requests a 15 to 20 pages long written report and an up to 2 pages long executive summary explaining why IS/IT Security is important to the company's core business, what is the reason and what are the implications of outsourcing it, and what would have to be done at Vattenfall in order to outsource.

- Assignment 2 - The CEO of Integralis requests the CISO of Integralis to prepare a qualitative risk analysis and a mitigation plan regarding the MSS business area and is expecting a 10 to 15 pages long written report and an up to 2 pages long executive summary on the subject.

\section{Outcome of the Study}

The team performance results were generally consistent between the two assignments with two apparent differences. One out of the seven teams did perform notably better on the second assignment while another team's performance slightly decreased in the long run. Although statistics are always subject to some variation and a number of various extraneous variables could have instigated the discrepancies, some of the possible explanations appeared to be more likely based on informal interviews and observations of the participants. The team that did better on the second assignment may have been hindered by inadequate ability of the leadership to coordinate their efforts in the early stages of group development [17]. The team that did worse may have had difficulty resolving internal conflict, reducing the team's productivity over time [18]. 
Below are the outcomes of the analysis of the team performance in correlation with the team profiles that were created with the results of each of the featured tests and across all the tests.

\subsection{Cognitive Reflection Test}

This test produced inconclusive results, mainly because the team that achieved best ranking on both assignments produced extraneous results. If we remove this team, the remaining teams did end up more or less where their profiles suggested that they would. The two most diverse teams being placed $2 \mathrm{~d}$ and $3 \mathrm{rd}$, and the less diverse teams falling behind in comparison.

Relatively high amount of highly reflective people (i.e. those who provide more accurate answers on the test) also seemed to correlate with superior performance of the team, which is not altogether surprising considering that, due to their expected time preferences, respondents who score high on the CRT are relatively more apt to planning, an essential aspect of risk management.

\subsection{Myths of Nature Test}

Many of the teams appeared to have comparable profiles in terms of diversity and balance based on the results of this test, making the test unable to explain the teams' ranking order in good detail. However, the only two teams that had full coverage of Myths preferences (i.e. 4 out of 4 possible preferences were present in these teams; most other teams had the coverage of $3 / 4$, and one team profile covering $2 / 4$ ) did in fact come 1 st and 2 nd respectively on both of the assignments. That is, this test was able to predict/explain the positions of the two leading teams in the study with their success being attributed to their superior diversity.

\subsection{Team Roles Test}

Diversity appeared to be a factor in the team's success, with higher being better; but also that certain functions in the team work can possibly have especial influence on the team's performance and neither a relative diversity nor good implementation of those functions may alone be enough to assure superior team performance, but a combination of both is required. This was not unanticipated, however.

Greater diversity is an asset when there are means to adequately coordinate it, in other cases it may actually become a liability. Belbin [5], for instance, stresses the importance of a good Coordinator in a successful team. Another team role that his research has shown to have special significance can be said to be analogous to the Brainstormer found in the typology employed in this study. Indeed, when these two team roles were regarded as having elevated importance compared to the other ones, this test's results were able to explain the exact order of all the teams on the ranking list with reasonable (given that statistics are not an exact science) accuracy and in more detail than the other two tests used in the study. 


\subsection{Across the Tests}

Curiously, despite having quite different frames of reference, the three tests generally did not disagree in their prognoses, with the one notable exception being the leading team's expected level of performance on the CRT as opposed by the other two tests. Although the results were not entirely conclusive, greater diversity of the teams did generally correlate with greater performance. The notion that a team should benefit from its composition being more balanced additionally to being more diverse could not be substantiated, however, due to the lack of evidence both for and against it.

The results of one team in particular stood out from the rest across all tests if one considers the context of this study. The team appeared to include fairly likeminded individuals who arguably possessed very favourable qualities for someone in a risk management position. More specifically, the members' answers on the CRT were, overall, very accurate, suggesting that they are not prone to making hasty decisions without first looking for possible errors in judgement and, due to the expected time preferences, are inclined towards planning. All but one of the team members chose Nature Perverse/Tolerant on the MNT, suggesting that they view nature as more or less predictable, and therefore consider that any changes in its state can (and as it is not entirely unbreakable, possibly should) be planned for. The TRT results are also the least diverse of all the teams, centring around the Thinker role (one half of the members have it as their highest score and the other half have it among their three highest ones), which specializes in analysis and planning, arguably the fundamental activities of risk management. This team has only achieved mediocre performance results on both assignments, further supporting the notion that the team's diversity may be instrumental in the team's success.

\section{Discussion on Implications of the Findings}

Looking back at the study's results, it is prudent to acknowledge certain limitations that it was subject to. One such limitation were the effects of individual qualities that could not be directly deduced from the results of the tests utilized (e.g. participants' relative dominance, cognitive ability, etc) on the team performance that were treated as extraneous variables in the experiment, which was done not by choice but rather due to limited availability of (human) resources and certain ethical issues (that, for instance, could arise if the participants' cognitive abilities were measured and then compared). Another evident limitation is, of course, the size of the sample, which is too small for any generalizations to be possible. One may, naturally, question how these limitations effect the accuracy and usefulness of the study results.

A simple answer is that the intention behind this study was not to provide an allinclusive investigation into the area in question in itself, but to determine whether such investigation would indeed be prudent and, if so, instigate further research into the subject matter. This moreover corresponds with the methodology selected for the study, which is essentially top-down, or deductive rather than inductive, allowing for potential research that builds on the study to add to the details. For a more elaborate answer, the application of the results of this study must be considered. From a purely academic perspective of (social) science, the more inclusive the approach the higher 
its value; however, from the standpoint of practicality and economic feasibility of real-world applications (including but not limited to the fields of security and risk management), there is always a matter of trade-off between the said feasibility and usability of the approach, and its complexity and comprehensiveness.

Bearing the above in mind, the implementation of the Team Roles model in this study was based on a reasonably simple hypothesis and yet has shown promising results. In addition, the Myths of Nature Test, while even simpler, has exhibited good potential in determining the leading teams in this study; if the test can be shown to produce such results consistently on a larger scale, it could be refined into a useful and practical (as well as cost-effective) tool devoid of unnecessary complexity. The two above mentioned tests can also be said to complement one another based on the study's outcome, which may create another possible dimension to their application. The Cognitive Reflection Test has produced inconclusive results, though, it may be too early to dismiss its usefulness in this context entirely for the same reason that it may be too early to draw any definitive conclusions about the other two tests based solely on the outcome of this study.

\section{References}

1. Foote, N., Matson, E., Weiss, L., Wenger, E.: Leveraging Group Knowledge for HighPerformance Decision-Making. Organizational Dynamics 31(3), 280-295 (2002)

2. Jackson, S.E., Joshi, A., Erhardt, N.L.: Recent Research on Team and Organizational Diversity: SWOT Analysis and Implications. Journal of Management 29(6), 801-830 (2003)

3. van Knippenberg, D., De Dreu, C.K.W., Homan, A.C.: Work Group Diversity and Group Performance: An Integrative Model and Research Agenda. Journal of Applied Psychology 89(6), 1008-1022 (2004)

4. Alberts, C.J., Dorofee, A.J.: Managing Information Security Risks: The OCTAVE Approach. Pearson Education, Boston (2003)

5. Belbin, R.M.: Management Teams: Why They Succeed or Fail, 2nd edn. Elsevier, Oxford (2004)

6. Margerison, C.J., McCann, D.J.: Team Management: Practical New Approaches. Management Books 2000, London (1995)

7. Frederick, S.: Cognitive Reflection and Decision Making. Journal of Economic Perspectives 19(4), 24-42 (2005)

8. Stanovich, K.E., West, R.F.: Individual Differences in Reasoning: Implications for the Rationality Debate? Behavioral and Brain Sciences 23(5), 645-726 (2000)

9. Kahneman, D., Tversky, A.: Prospect Theory: An Analysis of Decision Under Risk. Econometrica 47(2), 263-291 (1979)

10. McClure, S.M., Laibson, D.I., Loewenstein, G., Cohen, J.: Separate Neural Systems Value Immediate and Delayed Monetary Rewards. Science 306, 503-550 (2004)

11. Holling, C.S.: The Resilience of Terrestrial Ecosystems. In: Clark, C., Munn, R. (eds.) Sustainable Development of the Biosphere, pp. 292-317. Cambridge University Press, Cambridge (1986)

12. Timmerman, P.: Mythology and Surprise in the Sustainable Development of the Biosphere. In: Clark, C., Munn, R. (eds.) Sustainable Development of the Biosphere, pp. 436-453. Cambridge University Press, Cambridge (1986) 
13. Schwarz, M., Thompson, M.: Divided We Stand: Redefining Politics, Technology and Social Choice. Harvester Wheatsheaf, New York (1990)

14. Thompson, M., Ellis, R.J., Wildavsky, A.B.: Cultural Theory. Westview Press, Boulder (1990)

15. Plumeus: Compliance with APA's Standards for Psychological and Educational Testing (2006), http: / / www.psychtests.com/pdf / APA_Standards_Plumeus.pdf

16. Jung, C.G.: Psychological Types. In: Collected Works of C. G. Jung, 3rd edn., vol. 6, Princeton University Press, Princeton (1971) (Original work published 1921)

17. Wheelan, S.A.: Group Processes: A Developmental Perspective. Allyn \& Bacon, Boston (1994)

18. Hackman, J.R.: The Design of Work Teams. In: Lorsch, J. (ed.) Handbook of Organizational Behavior, pp. 315-342. Prentice-Hall, Englewood Cliffs (1987) 